\title{
Estudo Epidemiológico de fraturas faciais em uma sub-população brasileira
}

\author{
Epidemiological study of facial fractures in a brazilian subpopulation \\ Estudio epidemiológico de fracturas faciales en una subpoblación brasileña
}

Received: 04/11/2021 |Reviewed: 04/18/2021 |Accept: 04/21/2021 | Published: 05/07/2021

\author{
Marcus José da Hora Andrade \\ ORCID: https://orcid.org/0000-0003-1310-4688 \\ Faculdade de Ilhéus, Brasil \\ E-mail: m.andradeodontologia@gmail.com \\ Ana Grasiela da Silva Limoeiro \\ ORCID: https://orcid.org/0000-0003-4633-720X \\ Faculdade de Ilhéus, Brasil \\ E-mail: grasielalimoeiro@gmail.com \\ Caio Cesar Souza \\ ORCID: https://orcid.org/0000-0002-6445-5157 \\ ENDOBAHIA Cursos, Brasil \\ E-mail: caioitaobim@hotmail.com \\ Wayne Martins Nascimento \\ ORCID: https://orcid.org/0000-0003-4201-4710 \\ São Leopoldo Mandic, Brasil \\ E-mail: waynemartinsn@gmail.com \\ David Costa Moreira \\ ORCID: https://orcid.org/0000-0003-4720-541X \\ Faculdade de Ilhéus, Brasil \\ E-mail: dcmoreira@uesb.edu.br
}

\section{Resumo}

As lesões faciais, incluindo-se nestas as fraturas dos ossos que a compõem, assumem um papel de destaque nos atendimentos a pacientes politraumatizados nas emergências gerais, levando por muitas vezes a graves sequelas psicomotoras e sociais aos acometidos. Objetivo: Realizar um levantamento dos dados epidemiológicos de vítimas de traumas bucomaxilofaciais, atendidas no Hospital de base Luís Eduardo Magalhães em Itabuna/BA. Métodos: Foram utilizados 118 prontuários totalizando 147 fraturas. Os dados analizados foram: gênero, idade, cidade na qual o paciente reside, etiologia da fratura e sua localização, sendo tabulados, organizados e descritos com a utilização do programa Microsoft Excel e Word (versão 2016). Resultados: Observou-se uma predominância masculina (76,27\%), e a faixa etária mais prevalente foi entre 24 e 34 anos, sendo 32 a idade que mais se repertiu e 47,5 anos a média das idades. A mandíbula foi o osso mais afetado, o que representou $34,01 \%$, tendo os acidentes motociclísticos como principal etiologia e Itabuna a cidade com mais pacientes. Conclusão: Sendo os adultos jovens os pacientes mais afetados e os acidentes de trânsito, principalmente os motociclísticos, como principal etiologia, nota-se que tais fraturas poderiam ser evitadas com o incremento de mais medidas educativas no trânsito e uma maior punição aos motoristas imprudentes torna-se imprescincível na dimunuição de tal patologia.

Palavras-chave: Epidemiologia; Traumatismo facial; Fratura facial; Cirurgia bucomaxilofacial.

\begin{abstract}
Facial injuries, including bones fractures that comprise them, assume a prominent role in the care of polytrauma patients in general emergencies, often leading to severe psychomotor and social sequelae to those affected. Objective: To survey the victims' epidemiological data of oral and maxillofacial trauma treated at the Hospital de Base Luis Eduardo Magalhães in Itabuna / BA. Methods: A hundred and eight medical records were used, totaling 147 fractures. The analyzed data were gender, age, the city in which the patient resides, etiology of the fracture and its location, being tabulated, organized, and described using the Microsoft Excel and Word program (version 2016). Results: there was a male predominance (76.27\%); the most prevalent age group was between 24 and 34 years old, with 32 being the most repercussions and 47.5 years being the average age. The mandible was the most affected bone, representing $34.01 \%$, with motorcycle accidents as the primary etiology and Itabuna the city with the most patients. Conclusion: with young adults being the most affected patients and traffic accidents, especially motorcycles, as the primary
\end{abstract}


etiology, it is noted that such fractures could be avoided with the increase of more educational measures in traffic and greater punishment for reckless drivers makes it is essential in reducing such pathology.

Keywords: Epidemiology; Facial trauma; Facial fractures; Maxillofacial surgery.

\section{Resumen}

Las lesiones faciales, incluidas las fracturas de los huesos que las componen, asumen un papel destacado en la atención de los pacientes politraumatizados en las urgencias generales, provocando a menudo secuelas psicomotoras y sociales graves en los afectados. Objetivo: realizar un relevamiento de los datos epidemiológicos de las víctimas de trauma oral y maxilofacial atendidas en el Hospital de Base Luis Eduardo Magalhães en Itabuna / BA. Métodos: se utilizaron 118 historias clínicas, totalizando 147 fracturas. Los datos analizados fueron: sexo, edad, ciudad en la que reside el paciente, etiología de la fractura y su ubicación, siendo tabulados, organizados y descritos mediante el programa Microsoft Excel y Word (versión 2016). Resultados: Hubo predominio del sexo masculino (76,27\%), el grupo de edad más prevalente fue el de 24 a 34 años, siendo 32 la edad con mayor repercusión y 47,5 años la edad media. La mandíbula fue el hueso más afectado, que representó el 34,01\%, siendo los accidentes de motocicleta la principal etiología e Itabuna la ciudad con más pacientes. Conclusión: Siendo los adultos jóvenes los pacientes más afectados y los accidentes de tránsito, especialmente motocicletas, como principal etiología, se observa que tales fracturas podrían evitarse con el incremento de más medidas educativas en el tránsito y un mayor castigo a los conductores imprudentes hace imprescindible en reduciendo tal patología.

Palabras clave: Epidemiologia; Trauma facial; Fracturas faciales; Cirugía maxilofacial.

\section{Introdução}

As fraturas faciais têm um papel de destaque nos atendimentos a pacientes politraumatizados nas emergências gerais. Estudos realizados com a intenção de traçar o perfil epidemiológico dos traumatismos faciais em todo o mundo correlacionam as mudanças sociais, urbanas e rurais como agentes modificadores das relações interpessoais, gerando ações de violências física tanto de caráter pessoal como de grupo, sendo representado pelas agressões físicas, violência no trânsito, violência doméstica e violência à mulher (Falcão et al., 2009; Minari et al., 2020; De Macedo et al., 2020; Figueiredo et al., 2020).

A face é uma parte do corpo que está normalmente exposta sem proteções externas, que quando traumatizada resulta em lesões graves. Entende-se por fratura, a perda da continuidade de determinado osso por ruptura, sendo resultado de uma sobrecarga mecânica oriunda de golpes, forças ou trações capazes de superar a resistência óssea, gerando sua descontinuidade. O trauma facial é um assunto de grande relevância, pois é uma emergência cada vez mais frequente, estando associado ao aumento dos acidentes com veículos automotores e da violência urbana (Chalya et al., 2010; Minari et al., 2020; De Macedo et al., 2020; Figueiredo et al., 2020).

Uma agressão localizada na face não envolve apenas tecido mole e ossos, mas também, por extensão, pode acometer cérebro, olhos, seios da face e dentição. Quando um acidente que envolve maior energia cinética, outras lesões associadas e letais podem estar presentes e têm sido pouco relatadas (Wulkan et al., 2005). Segundo Eggensperger et al. (2006), as injúrias têm sido identificadas como a principal causa de redução de produtividade, incluindo menos anos de trabalho, em comparação às doenças do coração e ao câncer combinados.

Devido a variedade de pesquisas na referida área, bem como a necessidade de se conhecer as peculiaridades de cada região, o presente estudo objetivou traçar um perfil epidemiológico dos traumas bucomaxilofaciais dos pacientes atendidos no Serviço de Cirurgia e Traumatologia Bucomaxilofacial do Hospital de Base de Itabuna (BA), almejando alertar autoridades, cirurgiões bucomaxilofaciais e profissionais de saúde da importância da temática e incentivar campanhas de conscientização a cerca de evitar tais patologias. 


\section{Metodologia}

Baseado na metodologia utilizada em um artigo publicado recentemente (Minari et al. 2020), esta avaliação retrospectiva, realizada a partir da análise exploratória qualitativa e quantitativa, buscou analisar os traumas de face descritos nos prontuários dos pacientes atendidos no Serviço de Cirurgia e Traumatologia Bucomaxilofacial do Hospital de Base, na cidade de Itabuna durante o período de 1 de janeiro a 31 de dezembro de 2018.

Os dados foram coletados após da aprovação do Comitê de Ética do referido hospital, garantindo aporte legal para a pesquisa, proteção dos dados dos pacientes, e veracidade dos dados divulgados. A organização dos dados se deu por meio de um modelo de ficha, a qual continha as seguintes variáveis: sexo, idade, cidade em que o paciente reside, etiologia do trauma, local da fratura, esta subdividida em mandíbula, maxila , zigomático, ossos próprios do nariz (OPN) e completo naso-orbitoetmoidal (NOE). As etiologias foram organizadas da seguinte forma: agressão física, acidente automobilístico, acidente motociclístico, acidente ciclístico, queda da própria altura e projétil de arma de fogo (PAF).

Foram utilizados como critérios de exclusão os prontuários de pacientes que foram atendidos e não apresentavam fraturas nos ossos mencionados, pacientes que tinham outras doenças e anomalias que não fossem originadas das etiologias a serem consideradas e prontuários que não foram preenchidos com todos os dados necessários à pesquisa. Os dados foram organizados em tabelas do programa Microsoft Excel (versão 2016) e calculadas as quantidades, porcentagens, e medianas de algumas variáveis.

\section{Resultados}

Foram analisados 118 prontuários de pacientes com diagnóstico de trauma facial, nos quais foram constatadas 147 fraturas faciais. A Tabela 1 mostra que a maioria dos pacientes (90) eram do sexo masculino, representando a porcentagem de $76,27 \%$ dos casos e 28 do sexo feminino (23,73\%), chegando a proporção homem/mulher próxima de 3,2:1.

Tabela 1. Ocorrência de fratura relacionada ao gênero.

\begin{tabular}{|c|c|c|}
\hline Gênero & $\mathbf{N}$ & $\mathbf{\%}$ \\
\hline Masculino & 90 & 76,27 \\
\hline Feminino & 28 & 23,73 \\
\hline TOTAL & $\mathbf{1 1 8}$ & $\mathbf{1 0 0}$ \\
\hline
\end{tabular}

Fonte: Serviço de Cirurgia e Traumatologia Bucomaxilofacial do Hospital de Base de Itabuna

A faixa etária de maior prevalência foi de 24 a 34 anos (Tabela 2). A média de idade geral entre os pacientes foi de 47,5 anos, sendo 32 anos a moda, se repetindo 9 vezes (7,62\%). O paciente mais jovem atendido teve a idade de 13 anos e o mais velho 82 anos.

Tabela 2. Ocorrência de fraturas por faixa etária.

\begin{tabular}{|l|c|c|}
\hline Idades & $\mathbf{N}$ & $\mathbf{\%}$ \\
\hline $13-23$ anos & 22 & 18,64 \\
\hline $24-34$ anos & 41 & 34,74 \\
\hline $35-45$ anos & 26 & 22,03 \\
\hline $46-56$ anos & 13 & 11,01 \\
\hline 57-67 anos & 9 & 7,62 \\
\hline 68-82 anos* & 7 & 5,93 \\
\hline TOTAL & $\mathbf{1 1 8}$ & $\mathbf{1 0 0}$ \\
\hline
\end{tabular}

*Intervalo de 14 anos. Fonte: Serviço de Cirurgia e Traumatologia Bucomaxilofacial do Hospital de Base de Itabuna. 
Com relação às 147 fraturas, 50 (34,01\%) ocorreram na mandíbula, 40 (27,21\%) osso maxilar, 40 (27,21\%) no complexo zigomático; treze $(8,84 \%)$ nos ossos próprios nasais e quatro $(2,72 \%)$ no complexo naso-orbito-etmoidal, descritos na Tabela 3 .

Tabela 3. Distribuição quantitativa e percentual das fraturas por região anatômica.

\begin{tabular}{|l|c|c|}
\hline Tipo de fratura & N & \% \\
\hline Mandíbula & 50 & 34,01 \\
\hline Maxila & 40 & 27,21 \\
\hline Zigomático & 40 & 27,21 \\
\hline Ossos próprios do nariz & 13 & 8,84 \\
\hline Naso-orbito-etmoidal & 4 & 2,72 \\
\hline TOTAL & $\mathbf{1 4 7}$ & $\mathbf{1 0 0}$ \\
\hline
\end{tabular}

Fonte: Serviço de Cirurgia e Traumatologia Bucomaxilofacial do Hospital de Base de Itabuna.

Quanto aos agentes etiológicos foram registrados 38 casos (32,2\%) de acidentes motocilísticos; 29 casos de acidente automobilístico (24,57\%); 16 casos de PAF (projétil de arma de fogo) (13,55\%); 14 casos de queda da própria altura (11,86\%); 12 casos de agressão física $(10,16 \%)$ e nove casos de acidente ciclístico (7,62\%) (Tabela 4).

Tabela 4. Frequência e percentual das fraturas segundo o agente etiológico.

\begin{tabular}{|l|c|c|}
\hline Agente etiológico & Frequência & \% \\
\hline Acidente motociclistico & 38 & 32,20 \\
\hline Acidente automobilístico & 29 & 24,57 \\
\hline Acidente ciclístico & 09 & 7,62 \\
\hline Agressão física & 12 & 10,16 \\
\hline Projétil de arma de jogo & 16 & 13,55 \\
\hline Queda da própria altura & 14 & 11,86 \\
\hline TOTAL & $\mathbf{1 1 8}$ & $\mathbf{1 0 0}$ \\
\hline
\end{tabular}

Fonte: Serviço de Cirurgia e Traumatologia Bucomaxilofacial do Hospital de Base de Itabuna.

Os meses de janeiro e dezembro foram os que tiveram as maiores frequências de pacientes com fraturas faciais durante o ano de 2018, representando respectivamente 15 e 14 pacientes, equivalente a 12,71 e 11,86\%. Março, setembro e outubro são os meses com as menores frequências, já a média de atendimentos mensais foi de aproximadamente 9,83 pacientes por mês, dados descritos na Tabela 5 . 
Tabela 5. Distribuição mensal dos pacientes com fraturas durante o ano de 2018.

\begin{tabular}{|l|c|c|}
\hline MÊS & FREQUÊNCIA & \% \\
\hline JANEIRO & 15 & 12,71 \\
\hline FEVEREIRO & 10 & 8,47 \\
\hline MARÇO & 6 & 5,08 \\
\hline ABRIL & 9 & 7,62 \\
\hline MAIO & 10 & 8,47 \\
\hline JUNHO & 9 & 7,62 \\
\hline JULHO & 10 & 8,47 \\
\hline AGOSTO & 9 & 7,62 \\
\hline SETEMBRO & 8 & 6,77 \\
\hline OUTUBRO & 8 & 6,77 \\
\hline NOVEMBRO & 10 & 8,47 \\
\hline DEZEMBRO & 14 & 11,86 \\
\hline TOTAL & $\mathbf{1 1 8}$ & $\mathbf{1 0 0}$ \\
\hline
\end{tabular}

Fonte: Serviço de Cirurgia e Traumatologia Bucomaxilofacial do Hospital de Base de Itabuna.

Pacientes residentes da cidade de Itabuna (BA) foram os mais prevalentes na pesquisa (Tabela 6), representando $48,3 \%$ dos casos $(n=57)$. Ubatã $(5,08 \%)$, Valença $(5,08 \%)$ e Camamu $(5,08 \%)$ vieram em segundo lugar nessa estatística com 6 pacientes cada uma. Houve um total de trinta municípios de residência dos pacientes, porém, somente as quatro mais prevalentes foram descritas na presente pesquisa.

Tabela 6. Frequência e quantidade das cidades atuais dos pacientes.

\begin{tabular}{|l|c|c|}
\hline CIDADE & $\mathbf{N}$ & \% \\
\hline Itabuna & 57 & 48,3 \\
\hline Camamu & 6 & 5,08 \\
\hline Valença & 6 & 5,08 \\
\hline Ubatã & 6 & 5,08 \\
\hline Outras & 43 & 36,46 \\
\hline TOTAL & $\mathbf{1 1 8}$ & $\mathbf{1 0 0}$ \\
\hline
\end{tabular}

Fonte: Serviço de Cirurgia e Traumatologia Bucomaxilofacial do Hospital de Base de Itabuna.

\section{Discussão}

O Hospital de Base Luis Eduardo Magalhães, situado na cidade de Itabuna, no estado da Bahia, é referência no atendimento a diversas especialidades, dentre elas a Cirurgia e Traumatologia Bucomaxilofacial recebendo pacientes de cerca de 160 municípios, sendo sua localização estratégica por estar localizado próximo a rodovias que interligam diversas regiões do Estado.

A importância de estudos epidemiológicos para a elaboração de estratégias de prevenção e combate às mais diversas patologias são imensuráveis. Os dados que são coletados e analisados, sejam eles de qualquer área do conhecimento, ajudam a identificar as necessidades e peculiaridades de cada localidade ou região, obtendo assim medidas mais eficazes, tornando um sistema de saúde mais efetivo e com ações mais adequadas às particularidades regionais. Gomes et.al. (2005), afirmaram que a Epidemiologia tem em suas bases a clínica, a estatística e a medicina social. Essas origens múltiplas levam-na a ser a metodologia empregada em estudos comunitários, mas, também, clínicos. 
O desenvolvimento industrial do século XX propiciou aumento considerável da frota de veículos em circulação em todo o mundo. Os sistemas viários e o planejamento urbano, em geral, não conseguiram acompanhar o aumento do volume do tráfego. Desta maneira, a qualidade de vida, principalmente nas grandes cidades ficou prejudicada, contribuindo para o aumento da agressividade dos motoristas e para o crescimento da violência no trânsito, o que tem aumentado sensivelmente o número de acidentes com veículos automotores (Leporace et al., 2009; Minari et al., 2020; De Macedo et al., 2020; Figueiredo et al., 2020).

Para Freitas et al. (2009), o aumento na expectativa de vida e os avanços tecnológicos, observados na atualidade, provocaram uma mudança de comportamento nas pessoas, as quais passaram a adotar um estilo de vida mais ativo quando comparado com as décadas passadas. Esses fatores têm levado as pessoas a experimentarem maiores riscos, o que pode refletir em danos por traumatismos.

A Cirurgia e Traumatologia Bucomaxilofacial tem contribuído significativamente na assistência aos pacientes vítimas de traumatismo facial. A sua importância é observada nos grandes centros de atenção ao trauma em todo o mundo, sendo o cirurgião bucomaxilofacial bastante útil no diagnóstico e tratamento das lesões faciais. A sua atuação retoma tanto os tecidos moles quanto duros, destacando-se as correções das deformidades dento faciais (Bell, 2007; Minari et al., 2020; De Macedo et al., 2020; Minari et al., 2020; De Macedo et al., 2020; Figueiredo et al., 2020).

Pesquisas com traumatismos bucomaxilofaciais vêm ganhando destaque devido ao elevado índice de fraturas acometendo os ossos da face e suas sequelas. No presente estudo houve uma prevalência das lesões faciais em homens, representando estatisticamente 76,27\% das vítimas, onde a proporção homem/mulher ficou próximo de 3,2:1. Amarista et al., (2017) constataram as proporções homem/mulher de 2,7:1 e 6,1:1 respectivamente, dados que revelam uma maior susceptibilidade masculina à lesões e traumas, o que é justificado pelo acesso mais fácil dos homens a veículos, seja ele carro ou motocicleta, armas de fogo, álcool, e também a uma maior participação em brigas e agressões mediante comportamento violento.

Foi constatado através deste estudo que, adultos-jovens com idades entre 24 e 34 anos, constituem a faixa etária de maior prevalência, correspondendo a $34,74 \%$ do total. Isto se deve ao fato de que nesta faixa etária os indivíduos expõem-se mais aos fatores de risco, pois estão em plena atividade física e profissional. Tal constatação se assemelha ao de outros estudos, nos quais esse mesmo intervalo de idade também foi prevalente, equivalendo a 55\% de 521 (Pereira et al., 2011) e 32,4\% de 139 avaliados (Freitas et al., 2009). Mendes et al. (2016) realizaram um levantamento em serviço de saúde da rede pública de alta complexidade no município de Campina Grande/PB, onde foram registrados 54 casos de traumatismo facial nos meses de novembro e dezembro de 2012, dos quais 79,6\% eram homens, sendo as maiores frequências desses traumas ocorreram na segunda $(29,6 \%)$ e na terceira $(24,1 \%)$ décadas de vida, resultado próximo ao obtido por Baroni et al. (2019), onde em seu estudo verificou que a faixa etária mais afetada foi de 21 a 30 anos.

No presente estudo, os acidentes de trânsito foram divididos em - acidentes automobilísticos, ciclísticos e motociclísticos - dentre os quais os acidentes motocilisticos representaram o fator etiológico de maior incidência do estudo, representando 38 casos $(32,2 \%)$ do total. Ramos et al. (2018) realizaram um levantamento em hospital de referência da Paraíba e detectou que de todos os traumas bucomaxilofaciais entre janeiro de 2016 e dezembro de 2017, acidentes com motocicleta representaram 59,4\% do total. Thomson et al. (2003) verificaram que a maioria das fraturas faciais foram promovidas por quedas ao solo e Subhashraj et al. (2008) concluíram em seu estudo que o acidente motociclístico foi o principal fator etiológico. Entretanto há divergências no aspecto etiológico entre diversos autores, em decorrência principalmente do fator regional e cultural, como nos estudos de Júnior et al. (2010) que demonstravam que a agressão física foi o principal agente etiológico. 
Quanto ao tipo de fratura, o osso mais afetado foi a mandíbula representando 50 das 147 fraturas descritas, o equivale a 34,01\% dos casos. Pedroso Júnior et al. (2019) realizaram um estudo, onde a mandíbula foi o osso mais afetado, totalizando $33 \%$ dos casos, salientando que a mandíbula por ser o único osso móvel da face fica mais vulnerável a impactos e traumas. Charcanovic et al. (2004) realizaram um estudo em hospital em Belo Horizonte (MG) e verificou que a mandíbula também foi o principal osso acometido por fraturas, representando 51,9\% dos casos. O mesmo foi verificado por Mayrink et al. (2018) em estudo realizado em Vitória (ES), onde a mandíbula novamente foi o osso mais afetado por fraturas.

Os meses de Janeiro e Dezembro corresponderam aos maiores índices de ocorrências de fraturas, somados representam 24,57\% dos casos ( $\mathrm{n}=29$ ), tal índice muito provavelmente está atrelado ao verão e as férias, períodos onde há um aumento nas práticas de atividades físicas ao ar livre, elevação no fluxo de veículos nas estradas, maior consumo de bebidas alcoólicas e entorpecentes, inclusive entre motoristas, favorecendo aos crescentes índices de acidentes de trânsito e agressões físicas.

Em relação a procedência dos pacientes atendidos, a ampla maioria eram oriundos da cidade de Itabuna, revelando um índice 48,3\% (n=57), município com a maior população da região, segundo o IBGE (2019), com cerca de 213 mil habitantes, onde está localizado o hospital da pesquisa, via de passagem e acesso a importantes rodovias do Estado, pólo de serviços e entretenimento regional, possuindo casas noturnas e diversos bares contribuindo para um elevado índice de embriaguez e imprudências ao conduzir veículos motorizados elevando a quantidade de acidentes. Atrelado a esse fato, a cidade vem obtendo estatísticas negativas nas pesquisas de índices de violência, segundo dados do Atlas da Violência divulgado pelo IPEA em 2018, a cidade aparece entre as mais violentas do país para jovens.

Embora haja uma padronização na maioria dos estudos por uma prevalência de adultos jovens do sexo masculino acometidos por fraturas faciais, nota-se, um aumento de casos que envolvam mulheres, devido a principalmente a mudança de hábitos femininos neste século, correndo mais riscos a sofrerem traumas. Diante do trauma, a cirurgia corretiva revela ser o melhor método para que se reestabeleça a normalidade estética e funcional do traumatizado, fazendo com que o paciente retorne ao convívio social e suas atividades corriqueiras em menor tempo com menores ou nenhuma sequela.

\section{Conclusão}

Mediante análise epidemiológica dos resultados observou-se que os homens foram os mais afetados com fraturas faciais. A faixa etária mais incidente foi a de 24 e 34 anos, adultos-jovens com vida mais ativa e mais expostos a riscos. A mandibula foi o osso mais traumatizado, sendo os acidentes motociclísticos a etiologia mais comum. Concluímos por tanto que há uma necessidade clara e evidente de um maior incentivo por parte das autoridades competentes em ações de prevenção a acidentes de trânsito mediante um menor consumo de álcool, utilização de cinto de segurança e capacete a fim reduzir os traumas, principalmente os faciais oriundos de acidentes.

Novos estudos com maior número amostral e com um período mais longo de acompanhamento são necessários para fornecer dados epidemiológicos mais precisos, auxiliando na implementação de políticas públicas de prevenção ao traumatismo orofacial.

\section{Referências}

Amarista Rojas, F. J., Bordoy Soto, M. A., Cachazo, M., Dopazo, J. R., \& Vélez, H. (2017). The epidemiology of mandibular fractures in Caracas, Venezuela: Incidence and its combination patterns. Dental traumatology, 33(6), 427-432.

Baroni EJ, Celin RAT, Cunha SP. (2019). Etiology and incidence of facial fractures in a hospital located in a municipality in the extreme south of Santa Catarina state (Brazil). Journal of the Brazilian College of Oral and Maxillofacial, 5(1):22-29.

Bell, R. B. (2007). The role of oral and maxillofacial surgery in the trauma care center. Journal of oral and maxillofacial surgery, 65(12), $2544-2553$. 
Research, Society and Development, v. 10, n. 5, e27910514937, 2021

(CC BY 4.0) | ISSN 2525-3409 | DOI: http://dx.doi.org/10.33448/rsd-v10i5.14937

Chalya, P. L., Mabula, J. B., Ngayomela, I. H., Kanumba, E. S., Chandika, A. B., Giiti, G., ... \& Balamuka, D. (2010). Motorcycle injuries as an emerging public health problem in Mwanza City, Tanzania: A call for urgent intervention. Tanzania Journal of Health Research, 12(4), 214-221.

Chrcanovic, B. R., Freire-Maia, B., Souza, L. N. D., Araújo, V. D. O., \& Abreu, M. H. N. G. D. (2004). Fraturas de face: um estudo retrospectivo de 1 ano em um hospital de Belo Horizonte. Brazilian Oral Research, 18(4), 322-328.

De Macedo, D. S., Jacomo, T. S., Pimentel, A. C., Sendyk, W. R., \& Marão, H. F. (2020). Estudo epidemiológico retrospectivo das fraturas do complexo zigomático maxilar no Complexo Hospitalar Padre Bento em Guarulhos. Research, Society and Development, 9(8), e193985752-e193985752.

Eggensperger, N. M., Danz, J., Heinz, Z., \& Iizuka, T. (2006). Occupational maxillofacial fractures: a 3 -year survey in central Switzerland. Journal of oral and maxillofacial surgery, 64(2), 270-276.

Falcão, M. F. L., Leite Segundo, A. V., \& Silveira, M. M. F. D. (2005). Estudo epidemiológico de 1758 fraturas faciais tratadas no Hospital da Restauração, Recife/PE. Rev cir traumatol buco-maxilo-fac, 5(3), 65-72.

Figueiredo, C. M. B. F., Minari, I. S., Bonardi, J. P., Ponzoni, D., Brandini, D. A., \& Bassi, A. P. F. (2020). Perfil epidemiológico do trauma maxilofacial pediátrico: estudo retrospectivo de 20 anos de pacientes atendidos por serviço de pós-graduação universitária de Araçatuba, Brasil. Research, Society and Development, 9(8), e989986722-e989986722.

Freitas, D. A., Caldeira, L. V., Pereira, Z. M., Silva, A. D. M., Freitas, V. A. F., \& Antunes, S. L. N. O. (2009). Estudo epidemiológico das fraturas faciais ocorridas na cidade de Montes Claros/MG. Rev. bras. cir. cabeça pescoço, 113-115.

Gomes, P. P., Passeri, L. A., \& de Albergaria Barbosa, J. R. (2006). A 5-year retrospective study of zygomatico-orbital complex and zygomatic arch fractures in Sao Paulo State, Brazil. Journal of oral and maxillofacial surgery, 64(1), 63-67.

Junior, J. C. M., Keim, F. S., \& Helena, E. T. S. (2010). Aspectos epidemiológicos dos pacientes com traumas maxilofaciais operados no Hospital Geral de Blumenau, SC de 2004 a 2009. Arq. Int. Otorrinolaringol, 14(2), 192-198.

Leporace, A. A. F., Paulesini Júnior, W., Rapoport, A., \& Denardin, O. V. P. (2009). Estudo epidemiológico das fraturas mandibulares em hospital público da cidade de São Paulo. Revista do Colégio Brasileiro de Cirurgiões, 36(6), 472-477.

Mendes, N., Ferreira, B. C. D. B., Bracco, R., Martins, M. A. T., Fonseca, E. V. D., \& Souza, D. F. M. D. (2016). Perfil epidemiológico dos pacientes portadores de fraturas de face. Revista da Associacao Paulista de Cirurgioes Dentistas, 70(3), 328-329.

Minari, I. S., Figueiredo, C. M. B. F., de Oliveira, J. C. S., Brandini, D. A., \& Bassi, A. P. F. (2020). Incidência de múltiplas fraturas faciais: estudo retrospectivo de 20 anos. Research, Society and Development, 9(8), e327985347-e327985347.

Pedroso Júnior J.L; Vasques M.A.B; Moraes R.B, et al. (2019). Epidemiological study of facial injuries in Cacoal/RO, Brazil. J Braz Coll Oral Maxillofac Surg, 5(2):30-35.

Pereira, C. M., Silva Filho, M., Carneiro, D. S., Arcanjo, R. C., De Andrade, L. A., \& De Araújo, M. G. B. (2011). Epidemiology of maxillofacial injuries at a regional hospital in Goiania, Brazil, between 2008 and 2010. RSBO Revista Sul-Brasileira de Odontologia, 8(4), 381-385.

Ramos, J. C., Almeida, M. L. D. D., Alencar, Y. C. G. D., de Sousa Filho, L. F., Figueiredo, C. H. M. D. C., \& Almeida, M. S. C. (2018). Estudo epidemiológico do trauma bucomaxilofacial em um hospital de referência da Paraíba. Revista do Colégio Brasileiro de Cirurgióes, $45(6)$.

Subhashraj, K., Ramkumar, S., \& Ravindran, C. (2008). Pattern of mandibular fractures in Chennai, India. British journal of oral and maxillofacial surgery, 46(2), 126-127.

Thomson, W. M., Stephenson, S., Kieser, J. A., \& Langley, J. D. (2003). Dental and maxillofacial injuries among older New Zealanders during the 1990s. International journal of oral and maxillofacial surgery, 32(2), 201-205.

Wulkan, M., Parreira Jr, J. G., \& Botter, D. A. (2005). Epidemiologia do trauma facial. Revista da associação médica brasileira, 51 (5), 290-295. 